\title{
Ultrasound shear-wave elasticity and magnetic resonance diffusion coefficient show strong inverse correlation in small fibroadenomas
}

\author{
Nurten ANDAC BALTACIOGLU1 ${ }^{1}$, Derya TURELI² ${ }^{\text {D }}$ \\ ${ }^{1}$ Radiology Clinic, Vehbi Koc Vakfi American Hospital, Istanbul, Turkey. \\ ${ }^{2}$ Department of Radiology, School of Medicine, Marmara University, Istanbul, Turkey.
}

\author{
Corresponding Author: Derya TURELI \\ E-mail: deryatureli@yahoo.com
}

Submitted: 02.09.2020 Accepted: 08.11.2020

\begin{abstract}
Objective: Stiffness of breast lesions helps distinguish malignant from benign solid masses. Stiffness can be quantitatively measured by magnetic resonance and ultrasound imaging using apparent diffusion coefficient (ADC) and shear-wave elastography (SWE) techniques, respectively. This study aims to analyze correlations between SWE and ADC in biopsy-proven small fibroadenomas. Patients and Methods: Shear-wave elastography and ADC measurements of 50 fibroadenomas were evaluated retrospectively. Mean patient age was $41 \pm 13$ years (range 27-63). All lesions had maximum diameters of $\leq 20$ millimeters. Correlations between intralesional ADC, lesion-parenchyma ADC ratio, intralesional SWE, SWE heterogeneity index and lesion volume were analyzed.

Results: Mean values of lesions were as follows: $\mathrm{ADC}=1.71 \pm 0.22 \times 10-3 \mathrm{~mm} 2 / \mathrm{s}, \mathrm{ADC}$ ratio $=1.04 \pm 0.09$, maximum $\mathrm{SWE}=73.4 \pm 28.8$ $\mathrm{kPa}$, minimum $\mathrm{SWE}=43.9 \pm 21.8 \mathrm{kPa}$ and $\mathrm{SWE}$ heterogeneity index $=29.4 \pm 12.7 \mathrm{kPa}$. There was a strong inverse correlation between fibroadenoma ADC and SWE values (rho $=-0.746, \mathrm{p}<0.01$ ). Significant correlations were also found between fibroadenoma volume and ADC (rho $=-0.525, \mathrm{p}<0.05)$ and SWE $($ rho $=0.840, \mathrm{p}<0.01)$.

Conclusion: Apparent diffusion coefficient and SWE values show strong inverse correlation in small fibroadenomas. If proven threshold values for lesion characterization are revealed, ultrasonographic SWE and diffusion-weighted MRI have potential to be used interchangeably.

Keywords: Apparent diffusion coefficient, Fibroadenoma of breast, Magnetic resonance imaging, Ultrasound shear-wave elastography
\end{abstract}

\section{INTRODUCTION}

Stiffness of breast lesions helps distinguish malignant from benign solid masses. Tissue stiffness can be quantitatively and objectively assessed by magnetic resonance and ultrasound imaging using apparent diffusion coefficient (ADC) and shearwave elastography (SWE) techniques, respectively. Diffusionweighted magnetic resonance imaging (DW-MRI) is based on random diffusivity principle of water molecules. Diffusivity is impeded within dense and highly cellular tissues and can be quantitatively measured as ADC values. Ultrasound SWE, on the other hand, uses acoustic energy pulses to generate shear waves causing transient displacements in tissue which are then measured as shear moduli as absolute measures of tissue elasticity. Both techniques have previously been proven to be useful adjuncts in determining malignancy risk of large solid breast masses $[1,2]$. This study, on the other hand, aims to analyze correlations between SWE and ADC in a rather overlooked category of breast lesions, namely biopsy-proven small fibroadenomas.

\section{PATIENTS and METHODS}

Approval for the study was obtained from the Ethics Committee of Marmara University, School of Medicine with the decision numbered 2018.478. A total of 50 consecutive female patients with biopsy proven solitary fibroadenomas of maximum diameter $\leq 20$ millimeters within a one year period, from May 2018 till May 2019, were included after consenting to usage of relevant medical information in written form during follow-up clinical visits. Routine pre-biopsy diagnostic magnetic resonance and ultrasound studies were reviewed for ADC and SWE measurements.

Diagnostic breast MRI were realized using a 1.5 Tesla scanner (Optiva, General Electrics, USA) with the patient in prone position and both breasts in bilateral 16-channel phased array dedicated breast coils. DW-MRI examination was performed using a precontrast axial single-shot echo planar imaging (EPI) sequence with fat suppression and $b$ values of 0,400 , and $800 \mathrm{~s} / \mathrm{mm}^{2}$. TR/TE was $6055 / 69 \mathrm{~ms}$ and FOV was $350 \mathrm{~mm}$. A $3.5 \mathrm{~mm}$ slice thickness and

How to cite this article: Baltacioglu Andac N, Tureli D. Ultrasound shear-wave elasticity and magnetic resonance diffusion coefficient show strong inverse correlation in small fibroadenomas. Marmara Med J 2021;34(1):24-28. doi: 10.5472/marumj.866519 
a matrix of 128x96 was employed. ADC maps were automatically calculated by MRI scanner. ADC measurement of lesions were performed using specialized software (AW Volume Share, version 5, General Electrics, USA) by manually placing region of interest (ROI) on the largest cross-section of lesions (Figure 1). ADC values were recorded in units of $\mathrm{mm}^{2} / \mathrm{s}$ and control ADC measurements from contralateral breast parenchyma were also made in order to obtain a lesion to parenchyma ADC ratio.

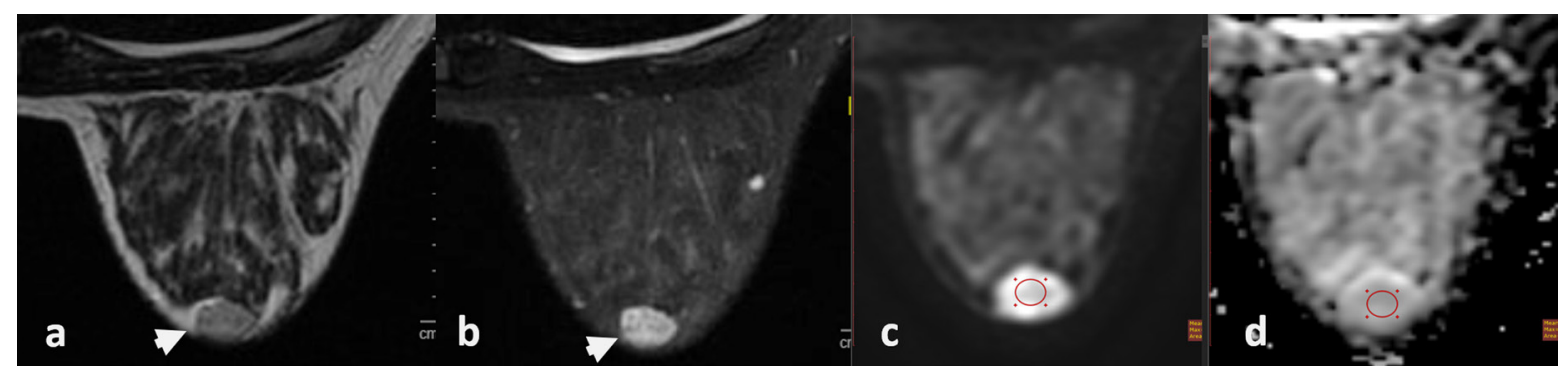

Figure 1. A 27-year-old female presenting with enlarging periareolar mass. (a) T2-weighted axial and (b) Fat-saturated T2-weighted axial images delineate a well-circumscribed oval retroareolar lesion (arrowheads). (c) Diffusion-weighted imaging and (d) corresponding Apparent Diffusion Coefficient maps show restricted water diffusion within the lesion. ADC measurements are performed using a free-hand elliptical ROI (ovals).

Pre-biopsy SWE of lesions was performed using a 12-16 $\mathrm{MHz}$ linear probe and a dedicated ultrasound system (Logic S8, General Electrics, USA) by a single operator who had over 15 years of experience in breast imaging and previous practice of SWE in different patient populations. Sonoelastographic measurements were made with the transducer held perpendicular to the skin while applying minimal pressure during one single breath-hold. A standard circular ROI of $2.25 \mathrm{~mm}^{2}$ area was used and intralesional maximum and minimum elasticity $\left(\mathrm{E}_{\max }\right.$ and $\left.\mathrm{E}_{\min }\right)$ were measured in units of kilopascals $(\mathrm{kPa})$ (Figure 2). Lesion volume was calculated using formula for spheroid volume, i.e. $\mathrm{V}=4 / 3 \times \pi \times \mathrm{r} 1 \times$ $\mathrm{r} 2 \times \mathrm{r} 3$; and SWE heterogeneity indices $(\mathrm{HI})$ were calculated according to formula $\mathrm{HI}=\mathrm{E}_{\max }-\mathrm{E}_{\min }$.

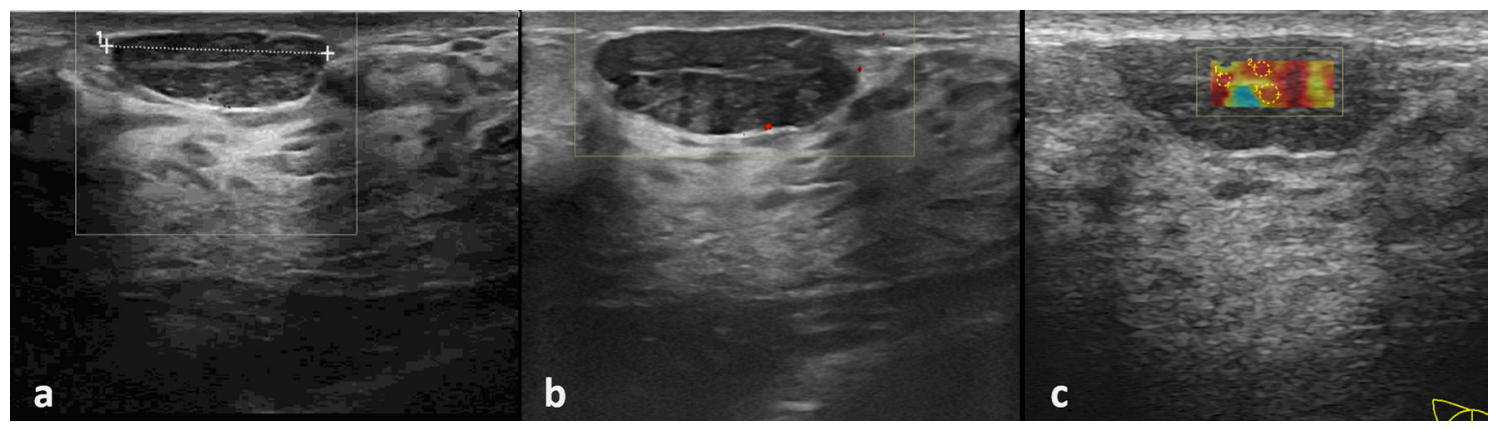

Figure 2. (a) Ultrasound image of retroareolar mass of the same patient. (b) Lesion had interval enlargement within one-year clinical follow-up, and was therefore sampled. Pathological diagnosis was consistent with fibroadenoma. (c) Shear-wave elastography of the lesion.

\section{Statistical Analyses}

Statistical analyses were performed using a commercially available software package (SPSS Advanced Statistics module, version 21.0, IBM, USA) and potential correlations among ADC measurements, i.e. intralesional ADC and lesion-to-parenchyma $\mathrm{ADC}$ ratio $\left(\mathrm{ADC}_{\text {ratio }}\right)$, and SWE values, namely $\mathrm{E}_{\max }, \mathrm{E}_{\text {min }}$ and HI, were sought. Correlation strength was categorized based on Spearman's correlation coefficient obtained from analyses (Table I). The Mann-Whitney U test was used to compare continuous data between groups. The results were evaluated at a $95 \%$ confidence interval. $\mathrm{p}<0.05$ was considered significant.
Table I. Strength of linear relationship corresponding to correlation coefficient value (Spearman's rho).

\begin{tabular}{|l|l|}
\hline Correlation coefficient value $(\mathbf{r h o}=)$ & Strength of correlation \\
\hline $0.80-0.99$ & Very Strong \\
\hline $0.60-0.79$ & Strong \\
\hline $0.40-0.59$ & Moderate \\
\hline $0.01-0.39$ & Poor \\
\hline
\end{tabular}

\section{RESULTS}

Patient ages ranged from 27 to 63 years (mean $41 \pm 13$ years). Mean lesion volume was $735 \mathrm{~mm}^{3}$ while median volume was 
$434 \mathrm{~mm}^{3}$ and volumes ranged from 113 to $3751 \mathrm{~mm}^{3}$. Mean ADC of lesions was $1.71 \pm 0.22 \times 10^{-3} \mathrm{~mm}^{2} / \mathrm{s}$ (range 1.37 to 2.11 $\mathrm{x} 10^{-3} \mathrm{~mm}^{2} / \mathrm{s}$ ). Mean lesion to normal parenchyma ADC ratio was $1.04 \pm 0.9$ and ranged from 0.90 to 1.21 . Mean elasticity measurements of lesions were as follows: $\mathrm{E}_{\max }=73.4 \pm 28.8 \mathrm{kPa}$ (range $41-133 \mathrm{kPa}), \mathrm{E}_{\min }=43.9 \pm 21.8 \mathrm{kPa}$ (range $\left.15-84 \mathrm{kPa}\right)$ and $\mathrm{HI}=29.4 \pm 12.7 \mathrm{kPa}$ (range 12-61 kPa).

A strong inverse correlation was found between $\mathrm{ADC}$ and $\mathrm{E}_{\mathrm{max}}$ of fibroadenomas ( $\mathrm{rho}=-0.746, \mathrm{p}<0.01$ ) (Figure 3 ). Additionally, there were a strong inverse correlation among mean ADC and $\mathrm{E}_{\text {min }}(\mathrm{rho}=-0.661, \mathrm{p}=0.003)$ and a moderate inverse correlation between $\mathrm{ADC}$ and $\mathrm{HI}$ (rho $=-0.538, \mathrm{p}=0.001$ ). $\mathrm{ADC}_{\text {ratio }}$ and $\mathrm{E}_{\max }$ had a moderate inverse correlation ( $\mathrm{rho}=-0.525, \mathrm{p}<0.01$ ) (Figure 4). There were no statistically significant correlations among $\mathrm{ADC}_{\text {ratio }}$ and $\mathrm{E}_{\min }$ or $\mathrm{HI}(\mathrm{rho}=-0.335, \mathrm{p}=0.013$ and rho $=$ $-0.248, \mathrm{p}=0.071$, respectively).

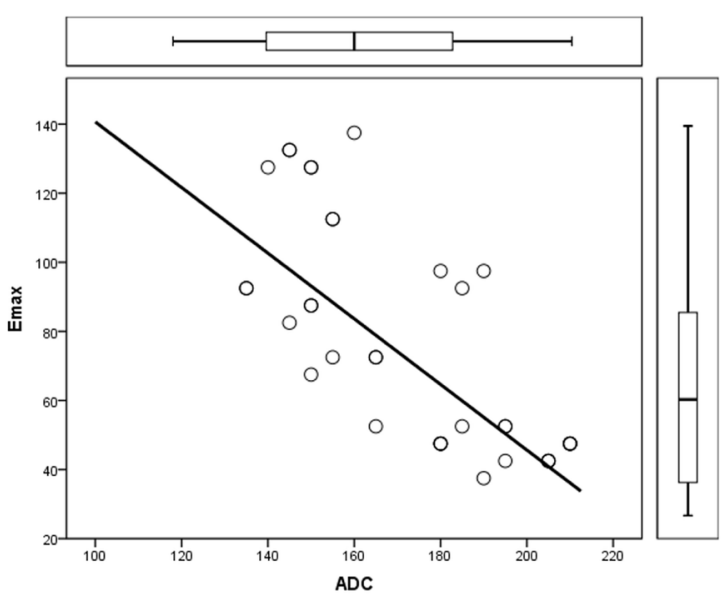

Figure 3. A strong inverse correlation was found between apparent diffusion coefficient (ADC) and maximal elasticity (Emax) of fibroadenomas (Spearman's rho $=-0.746, p<0.01$ ). ADC is presented in units of $\times 10^{-5} \mathrm{~mm}^{2} / \mathrm{s}$.

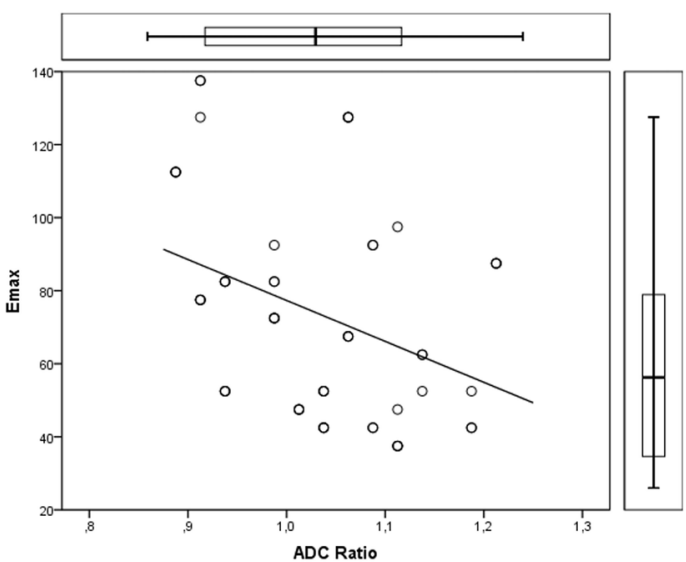

Figure 4. Lesion to parenchyma ratio of apparent diffusion coefficient $\left(A D C_{\text {ratio }}\right)$ and maximal elasticity $\left(E_{\max }\right)$ of fibroadenomas had a moderate inverse correlation (Spearman's rho $=-0.525, p<0.01) . E_{\max }$ is presented in units of $\mathrm{kPa}$.
ADC had a moderate inverse correlation with fibroadenoma volume (Spearmans rho $=-0.520, \mathrm{p}<0.001$ ) (Figure 5). There was no statistically significant correlations among $\mathrm{ADC}_{\text {ratio }}$ and fibroadenoma volume $(\mathrm{p}=0.12)$. On the other hand, fibroadenoma volumes were positively and strongly correlated with SWE measurements; Spearman's rho were 0.840, 0.680 and 0.745 for $\mathrm{E}_{\max }, \mathrm{E}_{\min }$ and $\mathrm{HI}$, respectively $(\mathrm{p}<0.001$ in all analyses $)$ (Figure 6).

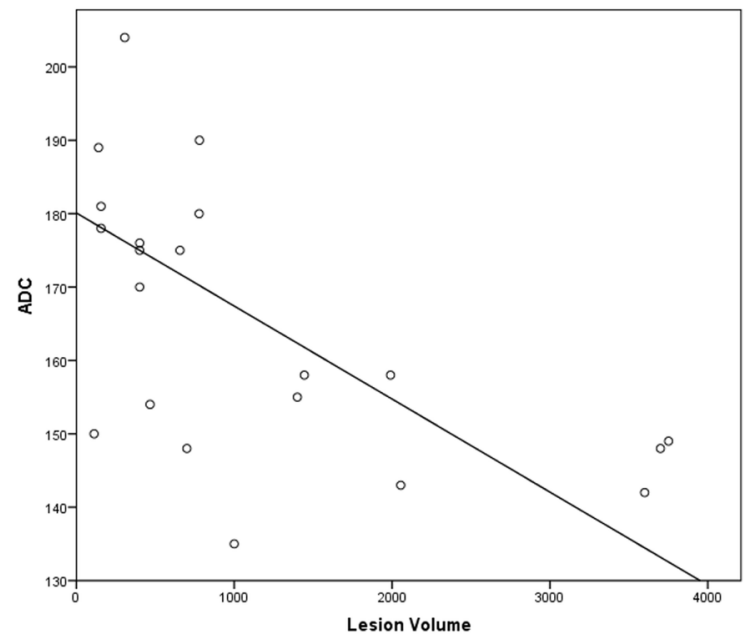

Figure 5. Apparent diffusion coefficient (ADC) showed a moderate inverse correlation with fibroadenoma volume (Spearman's rho $=-0.520$, $p<0.001)$. Volumes are presented in units of $\mathrm{mm}^{3}$ and $A D C$ in units of $x 10^{-5} \mathrm{~mm}^{2} / \mathrm{s}$.

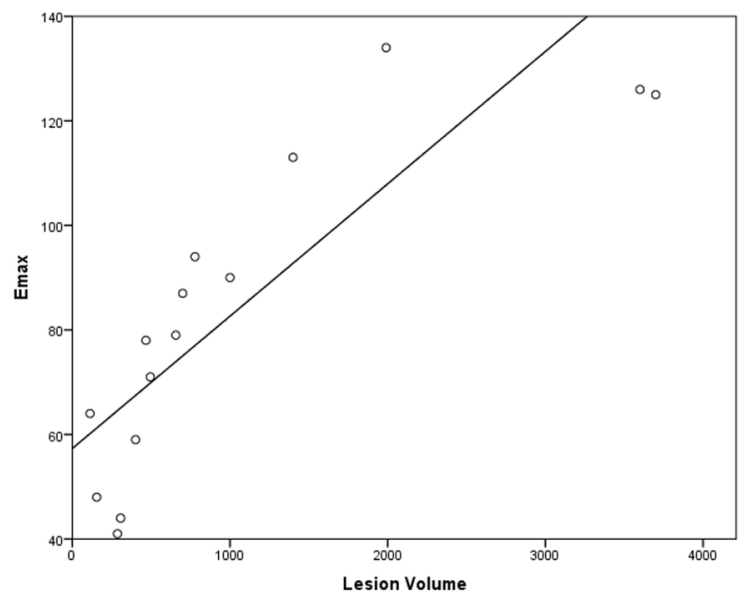

Figure 6. Lesion volumes were positively and strongly correlated with maximal elasticity (Emax) measured in fibroadenomas (Spearman's rho $=0.840, p<0.001)$. Volume is presented in units of $\mathrm{mm}^{3}$ whereas $E_{\max }$ is in units of $k P a$.

There was no statistically significant correlation between patient age and SWE or ADC measurements of fibroadenomas $(\mathrm{p}=0.23$ and $\mathrm{p}=0.47$, respectively). 


\section{DISCUSSION}

This study demonstrated a strong inverse correlation among ADC and SWE measurements of biopsy-proven small fibroadenomas, implying that ultrasound SWE has potential to be used interchangeably with diffusion-weighted MRI of such masses. ADC and SWE measurements also had significant correlation with lesion size, but not with patient age.

A previous study also showed significantly correlated lesion elasticity and ADC values. ADC values of malignant lesions was reported to be significantly lower than those of benign masses $\left(0.94\right.$ versus $\left.1.31 \times 10^{-3} \mathrm{~mm}^{2} / \mathrm{s}\right)$ and elasticity of lesions were also correlated with fibrosis grade histologically [3]. Our study population, comprising solely small biopsy-proven fibroadenomas, had comparable ADC measurements ranging from 1.37 to $2.11 \times 10^{-3} \mathrm{~mm}^{2} / \mathrm{s}$. There were no correlation between patient age and fibroadenoma stiffness in another study [4]. This was also concordant with results of our study.

In our study, lesion volume was significantly correlated with both ADC and SWE measurements. This was in accordance with a previous study which also demonstrated that size of tumor was correlated with its stiffness. In the mentioned study, mean elasticity of larger cancers, i.e. those with diameter $>15 \mathrm{~mm}$, was $167 \mathrm{kPa}$ whereas it was $109 \mathrm{kPa}$ for smaller malignancies [5]. In regard to fibroadenomas, a prior study proposed that major predictor of a lesion's stiffness was its size and thus different SWE thresholds were required for malignancy differentiation in lesions of differing sizes [4]. Another study proposed cutoff SWE values of 65 and $75 \mathrm{kPa}$ for small and larger lesions, respectively [6]. This proposal is, on the other hand, not in accord with our study in which maximal elasticity of small fibroadenomas ranged from 41 to $133 \mathrm{kPa}$ with a mean elasticity of $73.4 \pm 28.8 \mathrm{kPa}$.

SWE has proven to increase accurate characterization of lesions. In fact, stiffness of large and symptomatic breast masses are more easily assessed with SWE when compared to clinically occult lesions $[2,7]$. But, on the other hand, utility of SWE in assessment of malignity has certain caveats. Benign breast masses generally have lower SWE measurements than malignant lesions. But, still there is a wide grey-zone between these proposed cut-off values for benignity and malignancy. In a large cohort, SWE measurements of less than $80 \mathrm{kPa}$ indicated benignity with a specificity of $80 \%$ and $120 \mathrm{kPa}$ was proposed as malignancy threshold [8]. Furthermore, there is no widely accepted elasticity threshold for malignancy detection yet. Due to histologic characteristics of certain neoplasms, there is significant overlap of elasticity of malignant and benign lesions. Several studies have indeed proposed very different cut-off values for malignancy [5,9-11]. Most breast cancers are stiff and have mean elasticity over $50 \mathrm{kPa}$ at SWE. Cancers falsely cleared as benign by SWE are mostly small lesions, i.e. less than $10 \mathrm{~mm}$ in diameter, and low grade. Pure ductal carcinomas in situ are constitutionally softer than invasive cancers. Thus, a small and soft mass as shown by SWE may as well be an early cancer; so in such cases, it is advisable to use the biopsy option more liberally [12].
Another potential limitation SWE in differentiation of malignancy is that SWE may not be able to calculate lesion elasticity accurately in certain occasions. This occurs due to extremely diminished tissue deformation as seen in large and rigid infiltrative cancers which result in falsely low measurements because ultrasound cannot penetrate such highly attenuating scirrous tissues. This condition has potential to be confused as a low reading due to a lesion's benignity $[2,12]$. In such cases, diffusion MRI and ADC mapping is indeed more valuable.

In conclusion $\mathrm{ADC}$ and SWE measurements have strong inverse correlation in small fibroadenomas which implies, in select cases, a potential of ultrasonographic SWE to be used interchangeably with diffusion-weighted magnetic resonance imaging.

\section{Compliance with Ethical Standards}

Ethical Approval: Approval for the study was obtained from the Ethics Committee of Marmara University, School of Medicine with the decision numbered 2018.478. Written informed consent was obtained from all patients.

Financial Support: No disclosures.

Conflict of Interest: The authors declare that they have no conflicts of interest concerning this article.

Author contributions: Both authors were actively involved in data collection, analysis, and the writing of the manuscript.

\section{REFERENCES}

[1] Iima M, Honda M, Sigmund EE, Ohno Kishimoto A, Kataoka M, Togashi K. Diffusion MRI of the breast: Current status and future directions. J Magn Reson Imaging 2020;52:70-90. doi: 10.1002/jmri.26908.

[2] Barr RG. Sonographic breast elastography: a primer. J Ultrasound Med 2012;31:773-83. doi: 10.7863/ jum.2012.31.5.773.

[3] Matsubayashi RN, Imanishi M, Nakagawa S, et al. Breast ultrasound elastography and magnetic resonance imaging of fibrotic changes of breast disease: correlations between elastography findings and pathologic and short Tau inversion recovery imaging results, including the enhancement ratio and apparent diffusion coefficient. J Comput Assist Tomogr 2015;39:94-101. doi: 10.1097/RCT.000.000.0000000155.

[4] Elseedawy M, Whelehan P, Vinnicombe S, Thomson K, Evans A. Factors influencing the stiffness of fibroadenomas at shear wave elastography. Clin Radiol 2016;71:92-5. doi: 10.1016/j. crad.2015.10.029.

[5] Evans A, Whelehan P, Thomson K, et al. Quantitative shear wave ultrasound elastography: initial experience in solid breast masses. Breast Cancer Res 2010;12:R104. doi: 10.1186/ bcr2787.

[6] Skerl K, Vinnicombe S, Giannotti E, Thomson K, Evans A. Influence of region of interest size and ultrasound lesion size on the performance of $2 \mathrm{D}$ shear wave elastography (SWE) in solid breast masses. Clin Radiol 2015;70:1421-7. doi: 10.1016/j. crad.2015.08.010 
[7] Athanasiou A, Tardivon A, Tanter M, et al. Breast lesions: quantitative elastography with supersonic shear imagingpreliminary results. Radiology 2010;256:297-303. doi: 10.1148/radiol.10090385.

[8] Berg WA, Cosgrove DO, Doré CJ, et al. Shear-wave elastography improves the specificity of breast US: the BE1 multinational study of 939 masses. Radiology 2012;262:43549. doi: 10.1148/radiol.11110640.

[9] Tanter M, Bercoff J, Athanasiou A, et al. Quantitative assessment of breast lesion viscoelasticity: initial clinical results using supersonic shear imaging. Ultrasound Med Biol 2008;34:1373-86. doi: 10.1016/j.ultrasmedbio.2008.02.002.
[10] Tozaki M, Isobe S, Fukuma E. Preliminary study of ultrasonographic tissue quantification of the breast using the acoustic radiation force impulse (ARFI) technology. Eur J Radiol 2011;80:e182-7. doi: 10.1016/j.ejrad.2011.05.020.

[11] Meng W, Zhang G, Wu C, Wu G, Song Y, Lu Z. Preliminary results of acoustic radiation force impulse (ARFI) ultrasound imaging of breast lesions. Ultrasound Med Biol 2011;37:143643. doi: 10.1016/j.ultrasmedbio.2011.05.022.

[12] Vinnicombe SJ, Whelehan P, Thomson K, McLean D, Purdie CA, Jordan LB, et al. What are the characteristics of breast cancers misclassified as benign by quantitative ultrasound shear wave elastography? Eur Radiol 2014;24:921-6. doi: $10.1007 / \mathrm{s} 00330.013 .3079-4$. 